\title{
Rifampicin: biotransformation study using the fungus Cunninghamella elegans and monitoring through UHPLC-MS
}

\author{
Rafaela M. Sponchiado ${ }^{* a}$, Julia Sorrentino ${ }^{a}$, Leticia M. Cordenonsi ${ }^{\mathrm{a}}$, Alexandre M. Fuentefria ${ }^{\mathrm{a}}$, Martin \\ Steppe ${ }^{\mathrm{a}}$, Andreas Mendez ${ }^{\mathrm{a}}$, Cássia V. Garcia ${ }^{\mathrm{a}}$ \\ ${ }^{a}$ Programa de Pós-graduação em Ciências Farmacêuticas, Universidade Federal do Rio Grande do Sul, Av. Ipiranga \\ 2752, CEP 90610-000, Porto Alegre - RS, Brasil \\ *Corresponding author: rafasponchiado@hotmail.com
}

\begin{abstract}
Drug biotransformation studies appear as an alternative to pharmacological investigations of metabolites, development of new drug candidates with reduced investment and most efficient production. The objective of this study was to evaluate the capacity of biotransformation of Rifampicin (RIF) by the filamentous fungus Cunninghamella elegans as a microbial model of mammalian metabolism. In $120 \mathrm{~h}$, C. elegans transformed the drug into the following two metabolites: rifampicin quinone and novel metabolite. The products of rifampicin formed in vitro were monitored by HPLC-PDA, being identified through UHPLC-QTOF/MS. Metabolites were characterized according to their chromatographic profile, mass fragments and UV spectral data. The major metabolic pathways of rifampicin transformed by the fungus were oxidation, demethylation and mono-oxidation. The microbial transformation of RIF showed the potential of Cunninghamella species to produce RIF metabolites. This process can be used for a cost effective method for both known and unknown metabolite production.
\end{abstract}

Keywords: biotransformation; new metabolite; rifampicin; Cunninghamella elegans; UHPLC-MS. DOI: https://doi.org/10.22456/2527-2616.101989

\section{Introduction}

Rifampicin (RIF), a semisynthetic macrocyclic antibiotic, is widely used in the treatment of tuberculosis and staphylococcal infection. RIF acts by binding to the bacterial RNA (ribonucleic acid) polymerase which consequently blocks the synthesis of the bacterial RNA messenger (1). This drug has peculiar pharmacokinetic properties. RIF is well absorbed orally being widely distributed in body tissues and fluids. Its metabolism in humans occurs in the liver by the cytochrome (CYP) $\mathrm{P} 450$ resulting in the active metabolite 25desacetylrifampicin among other molecules $(2,3,4)$.

Microbial transformation has been used as a simple methodology for in vitro drug metabolism studies (5). Biotransformation using microorganisms offers the opportunity to help elucidating biosynthetic pathways, anticipate mammalian metabolism, and toxicity (6). Additionally, biotransformation reaction technology is considered to be an alternative for the development of sustainable technologies for the production of chemicals and drugs using principles of green chemistry (7). Biotransformation products can be labelled as 'natural' according to US and European (8).

Cunninghamella, a filamentous fungus, is considered one of the most important microorganisms for biotransformation studies since it can catalyze several reactions to various classes of compounds. Studies have demonstrated the ability of this fungus to metabolize a wide variety of drugs by mechanisms which are similar to what is observed during Phase I and II metabolism of mammals (9-12). Cunninghamella elegans carries at least one gene coding for a CYP enzyme closely related to the CYP51 family. These enzymes produce metabolites in amounts which allow the isolation of the same compounds generated by humans and animal metabolism. Besides, novel $C$. elegans metabolites presenting pharmacological interest also can be isolated $(13,14)$.

The aim of the present work was to evaluate the formation of metabolites of rifampicin using filamentous fungi Cunninghamella elegans ATCC 9245 through the use of robust analytical techniques with the ultra-high performance liquid chromatography-quadrupole timeof-flight mass spectrometry (UHPLC-QTOF/MS) for analysis and prediction of the chemical structure of the metabolites in the sample.

\section{Material and methods}

\section{Chemicals and reagents}

RIF (purity $\geq 95 \%$ ) was donated by Professor Myraci Muniz de Albulquerque (Pernambuco Federal University, Brazil). Rifampicin quinone (purity $\geq$ 99.99\%) United States Pharmacopeia (USP) reference standard was purchased from USP. Dichloromethane was purchased from Dinâmica (Diadema, Brazil) and $N, N$-dimethylformamide from Labsynth (Diadema, São 
Paulo). Methanol, acetonitrile, and ethanol of HPLC grade were purchased from Merck (New Jersey, USA), Vetec (São Paulo, Brazil), and Panreac (Barcelona, Spanish), respectively. Czapek-Dox broth and sabouraud dextrose agar were obtained from Kasvi (Paraná, Brazil). Water was purified by a Milli-Q system Millipore (Molsheim, France).

\section{Microbial Culture and Biotransformation Conditions}

Cunninghamella elegans ATCC 9245 was donated by the Bioconversion laboratory, Goiás Federal Univesity, Goiânia, Brazil. Stock cultures were cut in discs of 0.5 $\mathrm{cm}$ of diameter and maintained on sterile sodium chloride $0.9 \%(\mathrm{w} / \mathrm{v})$ at $5{ }^{\circ} \mathrm{C}$.

Spore solutions of $C$. elegans were prepared by growing the fungi for 7 days at $28{ }^{\circ} \mathrm{C}$ in tubes containing a sabouraud dextrose agar. Czapek-Dox liquid medium diluted to $50 \%$ (Sodium nitate $1.0 \mathrm{~g} / \mathrm{L}$, Magnesium Sulphate $0.25 \mathrm{~g} / \mathrm{L}$, Potassium Chloride $0.25 \mathrm{~g} / \mathrm{L}$, Di Potassium Phosphate $0.5 \mathrm{~g} / \mathrm{L}$, Ferrous Sulfate $0.005 \mathrm{~g} / \mathrm{L}$, and Sucrose $15 \mathrm{~g} / \mathrm{L}, \mathrm{pH} 7.0$ ), $100 \mathrm{ml}$ of this was measured with $10^{16}$ spores $\mathrm{mL}^{-1}$ of strain inoculated for 3 days at $28{ }^{\circ} \mathrm{C}$ in an orbital shaker (New Brunswick ${ }^{\mathrm{TM}}$ Innova $\left.^{\circledR} 2300\right)$ at $120 \mathrm{rpm}$. Then, the substrate $(250 \mathrm{mg})$, which was previously solved in $N, N$ dimethylformamide:ethanol $(1: 1 \mathrm{v} / \mathrm{v})$ was added into each flask to give the final RIF concentration of 500 $\mu \mathrm{g} / \mathrm{mL}$. The cultures were incubated at $28^{\circ} \mathrm{C}$, shaking at $120 \mathrm{rpm}$, for $120 \mathrm{~h}$. Samples $(2 \mathrm{~mL})$ of the liquid culture were periodically (1, 3, 4 and 5 days) withdrawn from each culture and extracted three times successively with dichloromethane $(4 \mathrm{~mL})$. Each extract was separately evaporated to dryness in exhaust hood at room temperature. The residues obtained were dissolved in 2 $\mathrm{mL}$ acetonitrile:water $(1: 1)$, filtered and $25 \mu \mathrm{L}$ (injection volume) analyzed by liquid chromatography. Control flasks consisted of culture broth without RIF (positive control: $\mathrm{C}+$ ), only sterile medium with RIF (negative control: C-), and culture broth (blank: W). Control flasks were included in the HPLC analysis.

\section{Instrumentation and HPLC conditions}

For characterization of metabolites, an HPLC method was first developed and validated (data nor shown) followed international guidelines (15). Liquid chromatographic analyses were conducted on a Shimadzu LC-10A system (Kyoto, Japan) equipped with an LC-20AT pump, SPD-20AV UV-VIS variable wavelength detector, DGU-20A5 degasser, CBM-20A controller system, and SIL-20A injection valve with 100 $\mu \mathrm{L}$ loop. Phenomenex C18 column (5 $\mu \mathrm{m}, 250 \mathrm{~mm}$ x 4.6 $\mathrm{mm}$ i.d.) and $\mathrm{C} 8$ guard column $(4 \times 3.0 \mathrm{~mm}$; Phenomenex, USA) were kept at $30{ }^{\circ} \mathrm{C}$ using a mobile phase consisting of a mixture of ammonium acetate $0.01 \mathrm{M}, \mathrm{pH} 4.0$ and acetonitrile $(50: 50, \mathrm{v} / \mathrm{v})$ at a flow rate of $1.0 \mathrm{~mL} \mathrm{~min} \mathrm{mith}^{-1}$ with detection at $254 \mathrm{~nm}$.

\section{Identification of metabolites: LC-QTOF MS analysis}

The structural elucidation of the metabolites formed was performed through the UHPLC-QTOF/MS system, UHPLC (Shimadzu-Nexera $\mathrm{x} 2$ ) equipped with a Shinpack XR ODS III column $(2.0 \mathrm{~mm} \times 50 \mathrm{~mm}, 1.6 \mu \mathrm{m})$ from Shimadzu coupled to the QTOF-MS mass analyzer (Impact II, Bruker Daltonics). The QTOF-MS system was equipped with an electrospray ionization (ESI) source, operating in positive ionization mode. The mobile phase consisted of acetonitrile and ammonium acetate $0,01 \mathrm{M} \mathrm{pH} 4.0$. The operation parameters of ESI were the following: capillary voltage, $4000 \mathrm{~V}$; end plate offset, $500 \mathrm{~V}$; nebulizer pressure, 4 bar (N2); drying gas, $0.28 \mathrm{~L} / \mathrm{min}(\mathrm{N} 2)$; and drying temperature, $200{ }^{\circ} \mathrm{C}$. The QTOF-MS system was operating in broadband collisioninduced dissociation (bbCID) acquisition mode and recorded spectra over the range $m / z 50-1000$ with a scan rate of $2 \mathrm{~Hz}$. A QTOF-MS external calibration was performed before each injection with a sodium formate solution. Data treatments were processed with Data Analysis 4.2 Software.

After $120 \mathrm{~h}$ of experiment, aliquots of biotransformation medium (drug, fungus, and medium: $\mathrm{BIO}$ ), $\mathrm{C}-, \mathrm{C}+$, and $\mathrm{W}$ were collected, extracted, and analyzed by UHPLCQTOF/MS.

\section{Results and Discussion}

Few information regarding the chromatographic analysis of RIF metabolites in vivo and in vitro are known. Passad and Singh (2009) studied the biotransformation of rifampicin in vitro (rat liver S9 fraction and microsomes) and in vivo (Sprague-Dawley rats). A total of 21 metabolites were identified, among them3formylrifamycin, rifampicin quinone and desacetylrifampicin $(17,18)$.

A representative HPLC-PDA chromatogram of rifampicin and its metabolites after 120 hours of transformation by C. elegans is shown in Fig. 1, which demonstrated the data from the biotransformation study, with the chromatogram (A) corresponding to the biotransformation medium (medium, fungus and drug), (B) negative control (medium and drug) (C) standard of the RIF quinone. The substrate controls showed there were no interference signals with the formed metabolites and the drug. The results of HPLC-PDA analyses indicated that RIF was metabolized in the biotransformation medium. It is possible to observe the RIF standard in $5.0 \mathrm{~min}$, and RIF quinone in $9.5 \mathrm{~min}$ (Figure 1, peak 2), which was confirmed by injection RIF quinone standard plus Czapek-Dox medium. Furthermore, in biotransformation medium (Fig 1A) another two peaks were detected, which can be related to possible metabolites (Fig 1; peaks 1 and 3). 


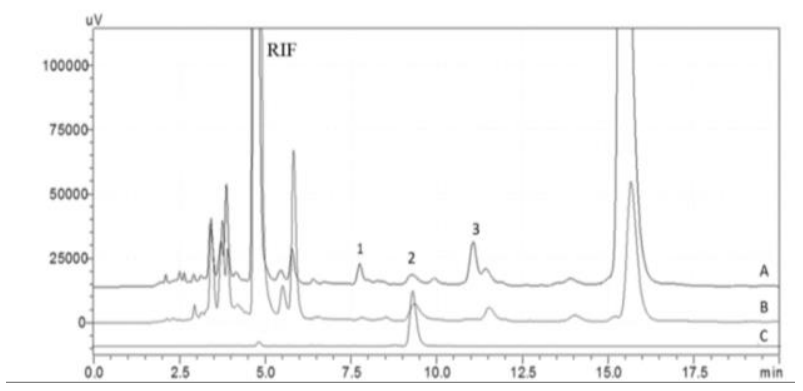

Figure 1: Chromatographic profile (HPLC-DAD) of reactional medium after 120h. (A) biotransformation medium (medium, drug and fungi), (B) negative control (medium and drug) and (C) standard RIF quinone. (1) and (3): possible metabolites. (2): RIF quinone.

After $120 \mathrm{~h}$ of incubation, being collected on day 1, 4 and 5 after adding the drug, it is possible to observe the reduction $(66.6 \%)$ of the drug concentration in relation to the negative control, which was submitted to the same extraction process. This behavior suggests drug consumption due to the action of the fungus and not only a process of drug oxidation caused by the culture medium. In this case, the fungus acts as a catalyst, accelerating the metabolization/degradation of RIF (Figure 2).

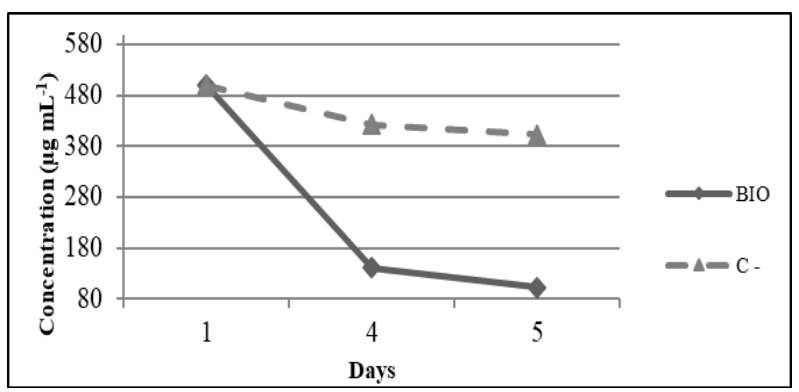

Figure 2: Decay representative graph of RIF concentration in 5 days of biotransformation, compared to the negative control in the same conditions. BIO (biotransformation medium); C(negative control).

The modern analytical techniques, in special hyphenated systems like UHPLC-QTOF/MS, are very useful to identify the biotransformation products even without previous treatment for separation of individual metabolites. Here in the present work, the samples were analyzed through UHPLC-QTOF/MS for elucidation of probable metabolites formed. The analytical conditions used were similar to the HPLC-DAD method and demonstrated good results. Analyzing the biotransformation medium (BIO) by UHPLC-QTOF/MS it is possible to detect the derivative rifampicin quinone ( $\mathrm{m} / \mathrm{z}$ 821.3962). Mass studies revealed the difference of two hydrogens (2.0157) in the structure, as compared to the drug standard. As illustrated on Figure 3, this metabolite is also detected in the negative control sample, but in a small intensity as compared to biotransformation medium (Fig 3A). As shown in Figure
3(B), mass fragmentation of RIF quinone resulted two stable fragments of $\mathrm{m} / z, 789.3694$ (N-demethylation) and 436.2324 (loss of aromatic and piperazine rings) (Figure 4). Other peaks were detected on zooming the spectra.

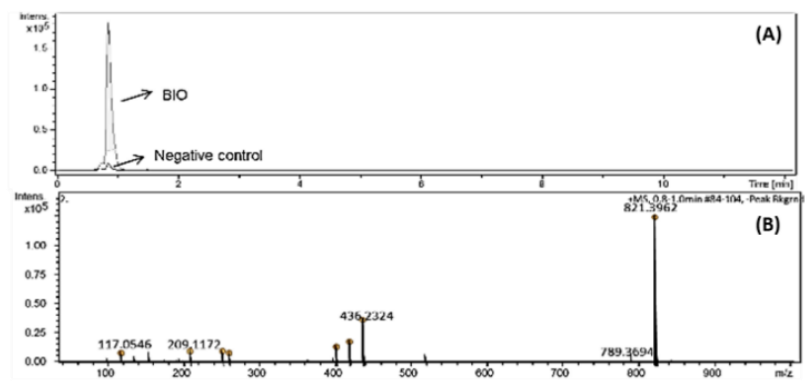

Figure 3: Comparative chromatograms of biotransformation medium (BIO) and negative control (A) and MS spectra of proposed metabolite, rifampicin quinone, in ESI mode positive (B).

Literature reports that the metabolite RIF quinone is formed in aqueous solutions favored by neutral and basic conditions, as well as naturally by oxidation (16). Then, RIF quinone is suggested as a degradation product whose formation is stimulated in the biotransformation medium, thus suggesting that the fungus used in this study accelerated the formation of the degradation product $(16,19,20)$. The degradation of an antibiotic favored by a filamentous fungus, which is found in the environment, is a positive point when the subject is bioremediation.

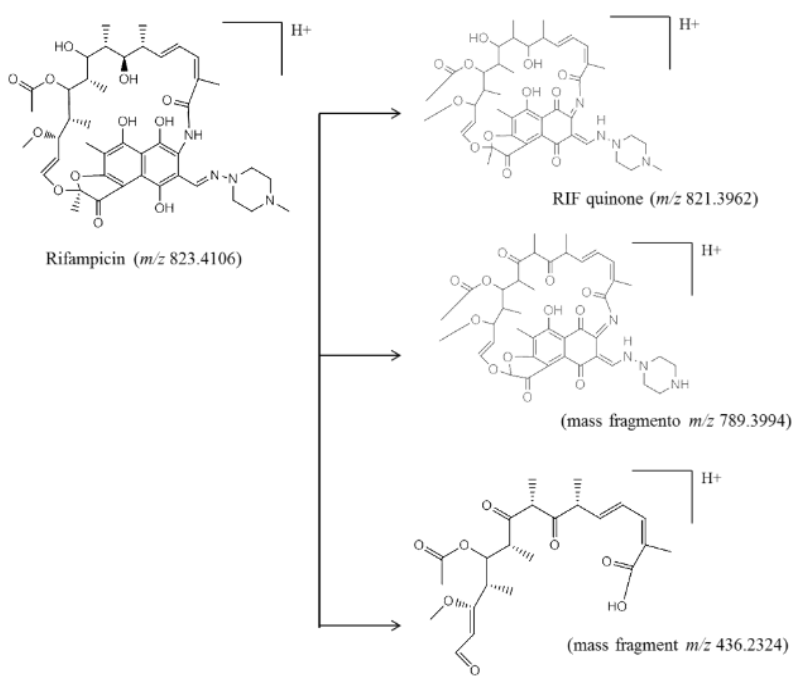

Figure 4: Proposed structures of RIF quinone and mass fragments obtained from UHPLC-QTOF/MS in ESI positive ion mode, after 120 hours of the biotransformation.

RIF can be metabolized either by hydrolysis to formylrifampicin or by deacetylation to form desacetyl rifampicin which retains practically all antibacterial activity. It was not possible to identify these known metabolites in the biotransformation medium, it was not possible to identify these metabolites already listed in the 
literature in the biotransformation medium, it is believed that by making some changes such as culture medium, agitation, concentration of the substrate (drug), inoculum or use of another microorganism, it could be detected in the study $(21,22)$.

Observing the chromatogram illustrated in the Figure 5 after 5 days' biotransformation study obtained from UHPLC-QTOF/MS analysis, the metabolite eluted at retention time of $10.0 \mathrm{~min}$ (Figure $5 \mathrm{~A}$ ) refers possibly to the peak detected in $11.5 \mathrm{~min}$ in HPLC-DAD analysis (Figure 1 peak 3 ). This product was found only in the biotransformation medium with $C$. elegans. The mass spectrum (Figure 5B) of this metabolite showed a molecular ion at $\mathrm{m} / \mathrm{z} 452.3217$ (Figure 5), with a loss of 370.6622 in reference to the molecular weight of rifampicin.

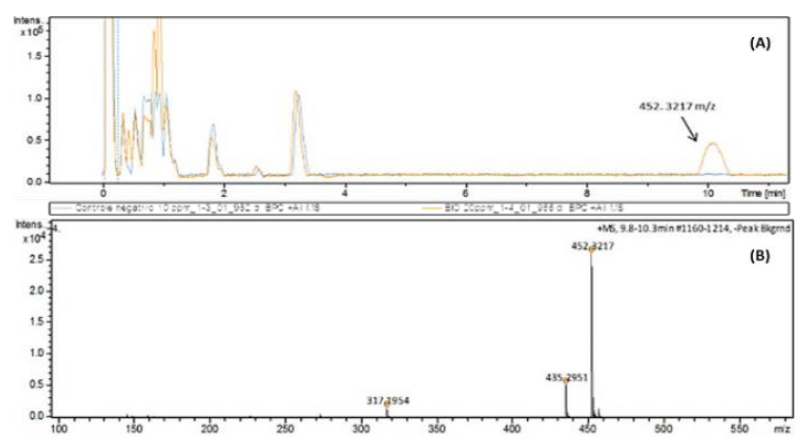

Figure 5: UHPLC-QTOF/MS chromatogram obtained from analysis of biotransformation medium in the study of rifampicin using Cunninghamella elegans. Chromatograms of overlapping biotransformation medium and negative control for comparison of the detected peaks (A), and mass spectrum of proposed metabolite ( $\mathrm{m} / \mathrm{z}$ 452.3217) (B).

The proposed molecular formula for this metabolite is $\mathrm{C}_{25} \mathrm{H}_{40} \mathrm{O}_{7}$ indicating the loss of eighteen carbons, five oxygen atoms and four nitrogens. It is suggested that the formation of this metabolite through ring cleavage, demethylation and loss of the piperazine fraction, which can be characterized as a RIF monoxygenation product $(\mathrm{m} / \mathrm{z}$ 451.1974) (Figure 6). For elucidation, our results were evaluated considering the study from Prasad and Singh (2009), which purposes a biotransformation pathway for rifampicin. Mono-oxygenation is mentioned as a way for metabolism and probably is involved to increase the drug polarity. In our case, the acetyl group is maintained intact, even being an important metabolic route, as reported by Prasad and Singh (2009). These authors have mentioned three metabolites formed by desacetylation: desacetylrifampicin, desacetyl-3formylrifampicin, and $\mathrm{N}$-demethyl-desacetylrifampicin. From our mass spectra results and possibilities for fragmentation, we have indicated a possible loss of hydroxyl group $(m / z, 434.1941)$ and desacetylation with other few modifications ( $m / z$ 317.1954) (Figure 6). This mono-oxygenated RIF metabolite with this purposed chemical structure is a novel compound, being described for the first time in the literature, even though Prasad and Singh (2009) report mono-oxygenation for RIF metabolism.

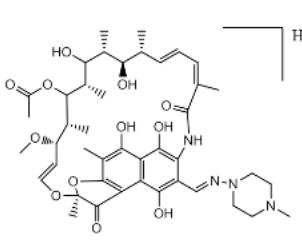

Rifampicin $(m / 2823.4106)$
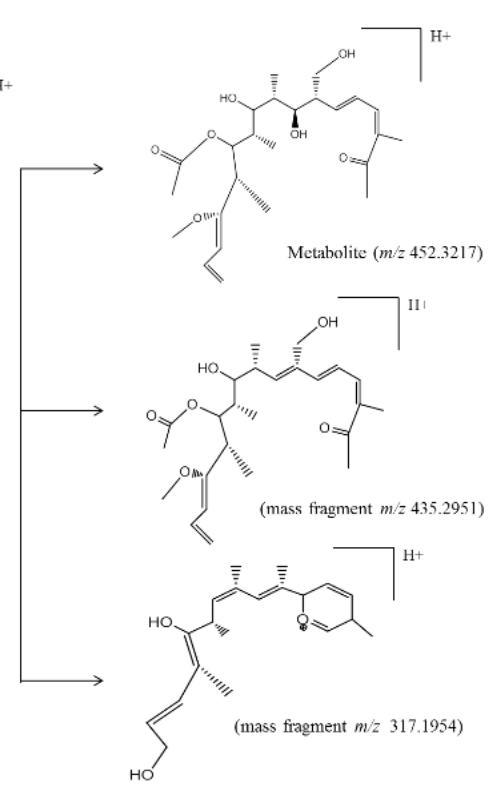

Figure 6: Proposed chemical structure of RIF metabolite (m/z 452.3217) obtained from biotransformation study with $\mathrm{C}$. elegans ATCC 9245.

\section{Conclusions}

In the present study, the microbial transformation of an important antibiotic with $C$. elegans was investigated. The results showed that rifampicin antibiotics were biotransfomed by fungi Cunninghamella elegans ATCC 9245 transforming a compound known as rifampicin quinone and generating another metabolite not yet reported in the literature. The structural elucidation of all metabolites were done based on mass fragmentation study combined with accurate mass measurements. In this study we observed great ability of $C$. elegans to metabolize rifampicin demonstrating that this microbial system represents an attractive alternative to the use of mammalian systems or chemical synthesis.

\section{Acknowledgements}

The authors are grateful to Coordenação de Aperfeiçoamento de Pessoal de Nível Superior (CAPES) for financial support and for granting research fellowships.

\section{Conflict of interest}

The authors declare no conflicts of interest. 


\section{References}

1. Zhang Y. The magic bullets and tuberculosis drug targets. Annu. Rev. Pharmacol Toxicol 2005; 45: 529-64.

2. Acocella G. Clinical pharmacokinetics of rifampicin. Clin Pharmacokinet 1978; 3: 108-127.

3. Drugbank, Rifampicin, 2017. Disponível em: https://www.drugbank.ca/drugs/DB01045 Acessed in 09.03.2019.

4. Mucklow JC. Martindale: The Complete Drug Reference. 36th Edition. London: Pharmaceutical Press, 2009.

5. Borges KB, Borges WDS, Pupo MT, Bonato PS. Endophytic fungi as models for the stereoselective biotransformation of thioridazine. Appl. Microbiol Biotechnol, 2007; 77: 669-674.

6. Adelin E, Servy C, Cortial S, Lévaique H, Gallard JF, Martin MT, et al. Biotransformation of natural compounds. Oxido-reduction of Sch-642305 by Aspergillus ochraceus ATCC 1009. Bioorg Med Chem Lett 2011; 21: 2456-2459.

7. Borges KB, De Souza Borges W, Durán-Patrón R, Pupo MT, Bonato PS, Collado IG. Stereoselective biotransformations using fungi as biocatalysts. Tetrahedron Lett 2009; 20: 385-397.

8. Molina G, Marostica MR, Pastore GM. Pseudomonas: A promising biocatalyst for the bioconversion of terpenes. Appl Microbiol Biotechnol 2013; 97: 1851-1864.

9. Asha S, Vidyavathi M. Cunninghamella - a microbial model for drug metabolism studies - a review. Biotechnol Adv 2009; 27: 16-29.

10. Srisailam K, Raj Kumar V, Veeresham C. Predicting drug interaction of Clopidogrel on microbial metabolism of Diclofenac. Appl Biochem Biotechnol 2010; 160: 1508-1516.

11. Barth T, Conti R, Puppo MT, Okano LT, Bonato PS. Chiral HPLC analysis of donepezil, 5-O-desmethyl donepezil and 6-O-desmethyl donepezil in culture medium: Application to fungal biotransformation studies. Anal Bioanal Chem 2012; 404: 257-266.

12. Quinn L, Dempsey R, Casey E, Kane A, Murphy CD. Production of drug metabolites by immobilised Cunninghamella elegans: from screening to scale up. J Ind Microbiol Biotechnol 2015; 42: 799-806.
13. Moody JD, Freeman JP, Fu PP, Cerniglia CE. Biotransformation of mirtazapine by Cunninghamella elegans. Drug Metab Dispos 2002; 30: 1274-1279.

14. Piska K, Żelaszczyk D, Jamrozik M, PEKALA E. Cunninghamella Biotransformation-Similarities to Human Drug Metabolism and Its Relevance for the Drug Discovery Process. Curr Drug Metab 2016; 17 : 107-117.

15. Food and Drug Administration. Guidance: Bioanalytical Method Validation. 2013.

16. Prasad B, Singh S. In vitro and in vivo investigation of metabolic fate of rifampicin using an optimized sample preparation approach and modern tools of liquid chromatography-mass spectrometry. J Pharm and Biomedical Anal 2009; 50: 475-490.

17. Balbão MS, Bertucci C, Bergamaschi MM, Queiroz RHC, Malfará WR, Dreossi SAC, et al. Rifampicin determination in plasma by stir bar-sorptive extraction and liquid chromatography. J Pharm and Biomedical Anal 2010; 51: 1078-1083.

18. Li W, Wang J, Yan ZY. Development of a sensitive and rapid method for rifampicin impurity analysis using supercritical fluid chromatography. J Pharm and Biomedical Anal 2015; 114: 341-347.

19. Li J, Zhu M, Rajamani S, Uversky VN, Fink AL. Rifampicin inhibits $\alpha$-synuclein fibrillation and disaggregates fibrils. Chem Biology 2004; 11: $1513-$ 1521.

20. Wang R, Cao WW, Khan AA, Cerniglia CE. Cloning, sequencing, and expression in Escherichia coli of a cytochrome $\mathrm{P} 450$ gene from Cunninghamella elegans. FEMS Microbiol Lett 2000; 188: 55-61.

21. Sorokoumova GM, Vostrikov VV, Selishcheva AA, Rogozhkina EA, Kalashnikova TYU, Shvets VI, et al. Bacteriostatic activity and decomposition products of rifampicin in aqueous solution and liposomal composition. Pharm Chem J 2008; 42: 35 38.

22. Becker C, Dressman JB, Junginger HE, Kopp S, Midha KK, Shah VP, et al. Biowaiver Monographs for Immediate Release Solid Oral Dosage Forms: Rifampicin. J Pharma Sci 2009; 98: 2252-2267. 